\title{
Polylactic acid and polyethylene glycol prevent surgical adhesions
}

\author{
Ozpolat B ${ }^{1}$, Gunal N¹, Pekcan Z², Ayva ES ${ }^{3}$, Bozdogan $\mathrm{O}^{4}$, Gunaydin $\mathrm{S}^{5}$, Dural $\mathrm{K}^{1}$ \\ Kirikkale University, School of Medicine, Department of Thoracic Surgery, Kirikkale, Turkey. \\ berkantozpolat@yahoo.com
}

\begin{abstract}
OBJECTIVES: Re-mediastinoscopy could be risky because of adhesions from the previous mediastinoscopy. The aim of this study was to evaluate the efficacy of a bio-resorbable barrier on adhesion formation in a remediastinoscopy rat model.

METHODS: Mediastinal dissection similar to mediastinoscopy was done in twenty-eight rats and a polymeric film comprising of polylactic acid and polyethylene glycol (Repel-cv ${ }^{\circledR}$, SyntheMed Inc., NJ, USA) was placed on trachea in the study groups. Group 1 (sham, sacrificed at day 30), Group 2 (single barrier, sacrificed at day 30), Group 3 (single barrier, sacrificed at day 60), Group 4(double layer barrier, sacrificed at day 60). Mediastinal adhesions, degree of inflammation, vascular proliferation, foreign body reaction and fibroblast proliferation was compared.

RESULTS: Macroscopic dissection showed significantly dense adhesions in Sham Group and Group 3 ( $p<$ 0.05). Histopathologic examination showed that there was a significant difference between groups when the foreign body reaction and fibroblast proliferation was evaluated $(p<0.05)$. No significant difference was present between the groups in terms of inflammation and vascular proliferation $(p>0.05)$.

CONCLUSIONS: This unique experimental study showed that adhesion barrier was effective as single layer application at day 30 and double layer application at day 60. At clinical conversion, by the application of barrier, the formation of adhesions might be decreased to provide a safe re-mediastinoscopy (Tab. 2, Fig. 4, Ref. 23). Text in PDF www.elis.sk.

KEY WORDS: mediastinoscopy, thoracic surgery, fibrosis, polylactic acid, polyethylene glycol.
\end{abstract}

\section{Introduction}

Re-mediastinoscopy is one of the repeat surgical interventions in thoracic surgery. The current indications of re-mediastinoscopy are the negative result or incomplete first mediastinoscopy, recurrent and second primary lung cancer, lung cancer occurring after a malignant disease, and restaging following induction therapy. The most frequent indication among them is restaging. The importance of restaging is clearly stated because only patients with proven mediastinal downstaging will benefit from a subsequent surgical resection (1-3).

The results of remediastinoscopy, however, may be disappointing because an incomplete or absent biopsies can be obtained due to fibrosis or adhesion formation in the previous mediastinoscopy (4). An insufficient staging up to $18 \%$ of the patients, and $0.7 \%$ morbidity due to severe hemorrhage was reported (5). Accordingly, while some authors were considering re-mediastinoscopy

${ }^{1}$ Kirikkale University, School of Medicine, Department of Thoracic Surgery, Kirikkale, Turkey, ${ }^{2}$ Kirikkale University, Faculty of Veterinary, Department of Surgery, Kirikkale, Turkey, ${ }^{3}$ Baskent University, School of Medicine, Department of Pathology, Ankara, Turkey, ${ }^{4}$ Ankara Numune Education and Research Hospital, Department of Pathology, Ankara, Turkey, and ${ }^{5}$ Adatip Hospital, Department of Cardiovascular Surgery, Sakarya, Turkey

Address for correspondence: B. Ozpolat, MD, Kirikkale Universitesi Tip Fakultesi Hastanesi, Yahsihan, 71100, Kirikkale, Turkey.

Phone: +904444071 as an invaluable tool for restaging after induction therapy, others defined new techniques to access the mediastinum $(3,6)$.

Currently, adhesion barriers were used in many surgical interventions and had become of an increasing interest because of their effectiveness. Unfortunately, experimental studies to reduce the adhesions after mediastinoscopy are limited to a single study in rats concluding anti- adhesive agents could reduce the difficulties during re-mediastinoscopy performed within a month (7). However, to the best of our knowledge, late results of a bioresorbable adhesion barriers to reduce mediastinal adhesions were not investigated.

Here we performed an experimental study to evaluate the efficacy of a bioresorbable barrier on adhesion formation at first and second month, and when it was used as single or double layer, in a re-mediastinoscopy rat model.

\section{Materials and methods}

Twenty-eight male Wistar albino rats (300-350 g, 4-6 months old) were used in this study. All animals received human care in compliance with the European Convention on Animal Care and the study protocol was approved by the Animal Ethics Committee of the University (Protocol number: 10/15).

Repel-cv ${ }^{\mathrm{R}}$ (SyntheMed Inc.,NJ, USA) is a bioabsorbable adhesion barrier, which reduces the severity of postoperative cardiac adhesions in pediatric patients. Its components are polylactic acid and polyethylene glycol. The polymers used in it have been used in 
Tab. 1. The scores of macroscopic and microscopic examination of groups.

\begin{tabular}{|c|c|c|c|c|c|}
\hline & $\begin{array}{l}\text { Group } 1 \\
\text { Sham }\end{array}$ & $\begin{array}{c}\text { Group } 2 \\
30 \text { days } 1 \text { layer }\end{array}$ & $\begin{array}{c}\text { Group } 3 \\
60 \text { days } 1 \text { layer }\end{array}$ & $\begin{array}{c}\text { Group } 4 \\
60 \text { days } 2 \text { layers }\end{array}$ & $\mathrm{p}$ \\
\hline \multicolumn{6}{|l|}{ Macroscopic } \\
\hline Pretracheal adhesions & 19.50 & 7.50 & 21.50 & 9.50 & $0.001^{\mathrm{a}}$ \\
\hline \multicolumn{6}{|l|}{ Microscopic } \\
\hline Degree of inflammation & 16.64 & 19.36 & 13.79 & 8.21 & 0.056 \\
\hline Vascular proliferation & 17.50 & 13.50 & 17.50 & 9.50 & 0.109 \\
\hline Foreign body reaction & 16.00 & 16.00 & 8.00 & 18.00 & $0.013^{\mathrm{a}}$ \\
\hline Fibroblast proliferation & 20.50 & 7.50 & 22.50 & 7.50 & $0.000^{\mathrm{a}}$ \\
\hline
\end{tabular}

Data were given as the mean ranks. The differences among the groups considering all variables were evaluated by Kruskal-Wallis test. When the p value from Kruskal-Wallis test was statistically significant, Mood median test was used to determine the group that caused the difference.

a The difference was significant $(\mathrm{p}<0.05)$

Tab. 2. Additional statistical analysis for macroscopic adhesion and histopathologic markers.

\begin{tabular}{|c|c|c|c|c|c|c|c|c|}
\hline \multirow[b]{2}{*}{ Macroscopic adhesion } & \multirow{2}{*}{$\begin{array}{c}\text { Groups } \\
1\end{array}$} & \multirow{2}{*}{$\begin{array}{c}\text { Mean } \\
2.00\end{array}$} & \multirow{2}{*}{$\begin{array}{c}\text { St. Dev. } \\
0.816\end{array}$} & \multirow{2}{*}{$\begin{array}{l}\text { St. Er. } \\
0.309\end{array}$} & \multicolumn{2}{|c|}{$\begin{array}{c}\text { 95\% C.I. for } \\
\text { mean }\end{array}$} & \multirow{2}{*}{$\begin{array}{c}\text { Min } \\
1\end{array}$} & \multirow{2}{*}{$\frac{\operatorname{Max}}{3}$} \\
\hline & & & & & 1.24 & 2.76 & & \\
\hline & 2 & 0.29 & 0.488 & 0.184 & -0.17 & 0.74 & 0 & 1 \\
\hline & 3 & 2.29 & 0.488 & 0.184 & 1.83 & 2.74 & 2 & 3 \\
\hline & 4 & 0.57 & 0.976 & 0.369 & -0.33 & 1.47 & 0 & 2 \\
\hline \multirow[t]{4}{*}{ Degree of inflammation } & 1 & 1.86 & 1.464 & 0.553 & 0.50 & 3.21 & 0 & 3 \\
\hline & 2 & 2.29 & 0.756 & 0.286 & 1.59 & 2.98 & 1 & 3 \\
\hline & 3 & 1.57 & 0.535 & 0.202 & 1.08 & 2.07 & 1 & 2 \\
\hline & 4 & 0.71 & 0.951 & 0.360 & -0.17 & 1.59 & 0 & 2 \\
\hline \multirow[t]{4}{*}{ Vascular proliferation } & 1 & 0.71 & 0.488 & 0.184 & 0.26 & 1.17 & 0 & 1 \\
\hline & 2 & 0.43 & 0.535 & 0.202 & -0.07 & 0.92 & 0 & 1 \\
\hline & 3 & 0.71 & 0.488 & 0.184 & 0.26 & 1.17 & 0 & 1 \\
\hline & 4 & 0.14 & 0.378 & 0.143 & -0.21 & 0.49 & 0 & 1 \\
\hline \multirow[t]{4}{*}{ Foreign body reaction } & 1 & 0.86 & 0.378 & 0.143 & 0.51 & 1.21 & 0 & 1 \\
\hline & 2 & 0.86 & 0.378 & 0.143 & 0.51 & 1.21 & 0 & 1 \\
\hline & 3 & 0.29 & 0.488 & 0.184 & -0.17 & 0.74 & 0 & 1 \\
\hline & 4 & 1.00 & 0.000 & 0.000 & 1.00 & 1.00 & 1 & 1 \\
\hline \multirow[t]{4}{*}{ Fibroblast proliferation } & 1 & 1.71 & 0.756 & 0.286 & 1.02 & 2.41 & 1 & 3 \\
\hline & 2 & 0.00 & 0.000 & 0.000 & 0.00 & 0.00 & 0 & 0 \\
\hline & 3 & 2.14 & 0.900 & 0.340 & 1.31 & 2.97 & 1 & 3 \\
\hline & 4 & 0.00 & 0.000 & 0.000 & 0.00 & 0.00 & 0 & 0 \\
\hline
\end{tabular}

St. Dev. - Standard deviation, St. Er. - Standard error, C.I. - Confidence interval, Min - minimum, Max - maximum

many medical implants and defined to be safe and biocompatible. The thickness is 137 microns. It does not require placement on a dry surgical area. The polymers degrade after exposure to moisture and are eliminated from the body in approximately one month.

Rats were divided into the four groups as; Group 1 (Mediastinal dissection, sacrificed at day 30, n=7), Group 2 (Mediastinal dissection, single barrier applied group, sacrificed at day 30, $\mathrm{n}=$ 7), Group 3 (Mediastinal dissection, single barrier applied group, sacrificed at day 60, $n=7$ ), Group 4 (Mediastinal dissection and double layer barrier applied group, sacrificed at day 60, $n=7$ ).

\section{Surgery}

Rats were anesthetized with $50 \mathrm{mg} / \mathrm{kg}$ ketamine hydrochloride plus $10 \mathrm{mg} / \mathrm{kg}$ xylazine hydrochloride administered intramuscularly. Under sterile conditions, a mid-cervical horizontal skin incision was made over the trachea. In the Group 1 (Sham group), upper mediastinal dissection was done, i.e. the strap muscles were divided and retracted laterally, by the use of a mosquito clamp the pretracheal region was prepared to access the paratracheal area and a dissection was extended to the subcarinal region. Gaze abrasion of the mediastinoscopy tract was performed and numerous bleedings were created, than they were tamponated by a sponge for hemostasis. In the Groups 2 and 3 after a similar dissection, Repel- $\mathrm{cv}^{\mathrm{R}}$ was prepared in adequate size, soaked in saline solution for approximately two minutes and then placed and fixed in the pocket that was created previously. In the Group 4 after mediastinal dissection and similar preparations, the barrier was bended and applied as double layer. The strap muscles and skin were closed sequentially.

\section{Autopsy}

Autopsies were performed on day 30 in the Groups 1, 2 and on day 60 in the Groups 3 and 4 under a general anesthesia.

\section{Macroscopy and microscopy}

Trachea was totally removed without compromising strap muscle integrity and evaluated for adhesion formation.

Correctly oriented sections of the dissection site and nearby trachea were taken and placed in $10 \%$ buffered formalin. After the tissues were embedded in paraffin, slices of 4-6 microns were prepared and stained with Hematoxylin-Eosin. Masson Trichrome stain was also used to evaluate fibrosis. 

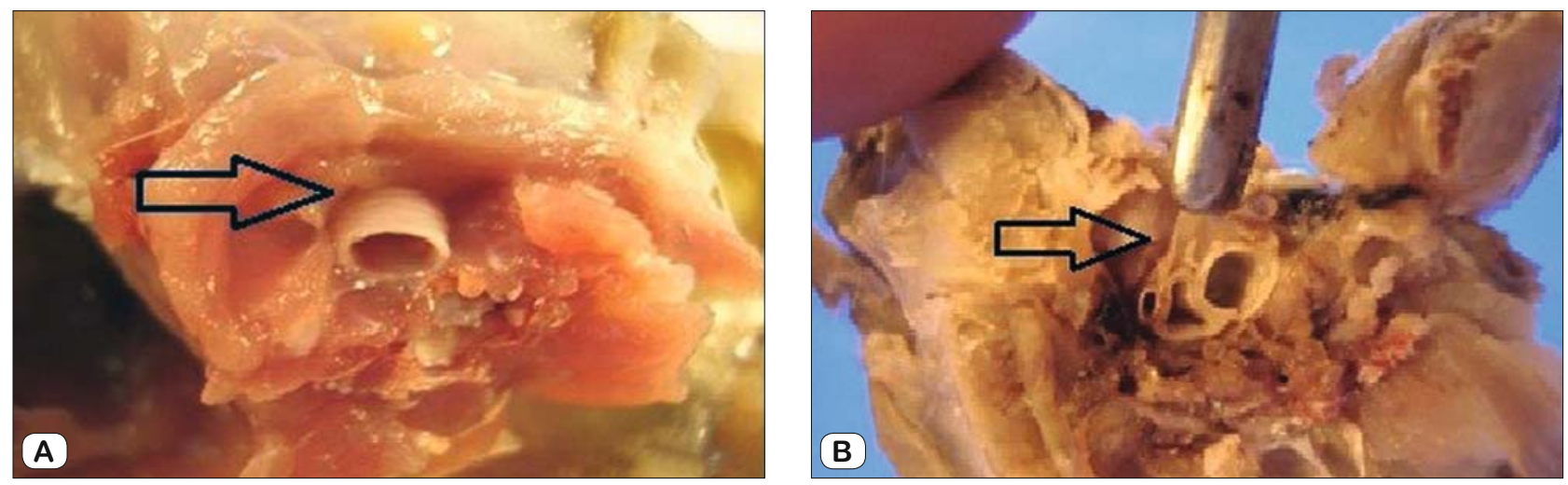

Fig. 1. Macroscopic appearance of paratracheal region (a) shows that anterior and lateral tracheal region is free of adhesions, (b) shows the dense adhesions at the same region in sham group.

Macroscopic and histopathologic analysis on different aspects was done by two pathologists who were blinded to the groups. Macroscopic adhesions were graded as: grade 0 , null; grade 1 , mild; grade 2 , moderate and grade 3 , severe. The degree of inflammation, vascular proliferation, foreign body reaction and fibroblasts were evaluated and scored. The degree of inflammation was graded as: grade 0 , null; grade 1 , mild; grade 2 , moderate and grade3, severe. If vascular proliferation was absent it's graded as 0 , if present it's graded as 1 . If foreign body reaction was absent it's graded as 0 , if present it's graded as 1 . Fibroblast proliferation was graded as: grade 0 , null; grade 1 , mild; grade 2 , moderate and grade 3 , extensive (7).

\section{Statistical analysis}

Data analysis was performed by using the SPSS for Windows, version 15.0 (SPSS Inc., Chicago, IL, United States) and MINITAB version 16. The differences among the groups considering all variables were evaluated by Kruskal-Wallis test. When the p value from Kruskal-Wallis test was statistically significant, Mood median test was used to determine the group that caused the difference. The p value less than 0.05 was considered as statistically significant.

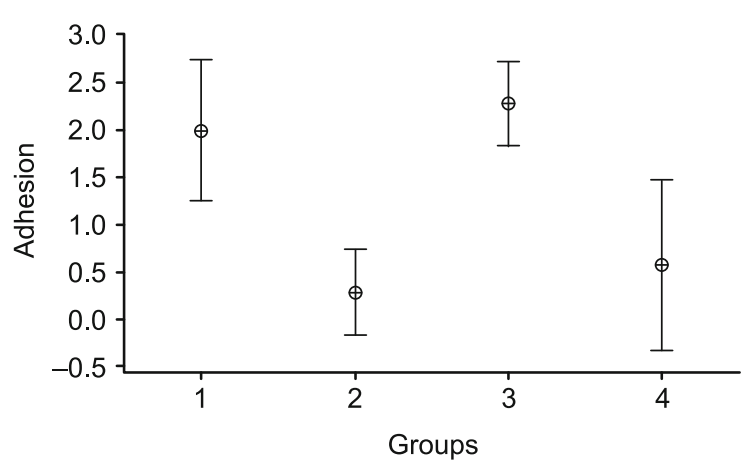

Fig. 2. Interval plot of macroscopy. In Groups 2 and 4, adhesions were significantly less than other Groups.
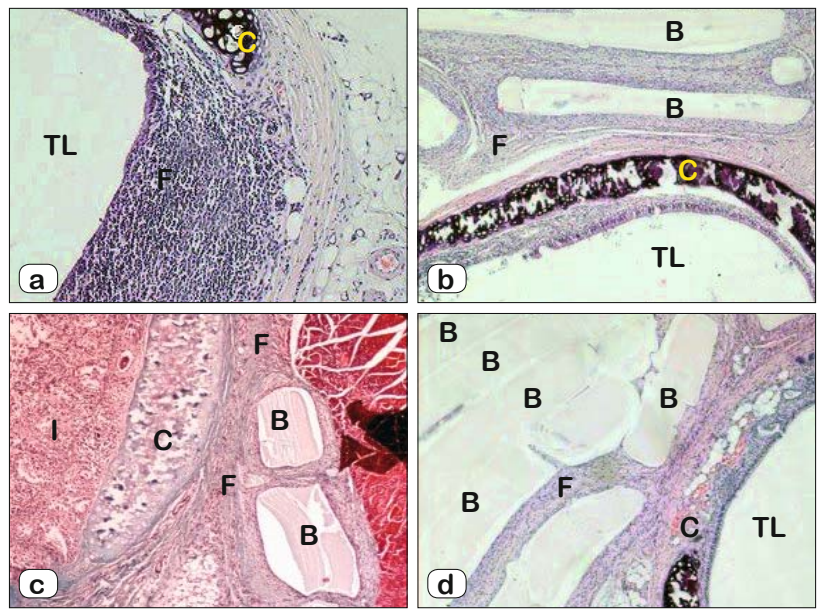

Fig. 3. Microscopic section of trachea and mediastinum. a) Massive fibrosis at sham group where no barrier was used. Hematoxylin and Eosin, X 40. b) Minimal fibrosis formation around membrane at 30 day scarification and single layer barrier used group. Hematoxylin and Eosin, $X$ 40. c) Massive fibrosis around membrane and chronic inflammation at group 3 and collagen fibers encircling foreign body granulomas. Masson Trichrome, $X$ 40. d) Extensive degradation of double layered membrane around trachea. Hematoxylin and Eosin, X 40. TL - Tracheal lumen, F - Fibroblast, C - Cartilage, B - Barrier, I - Inflammation.

\section{Results}

Starting from awakening period no problems were observed in any rats and none of them died. The scores of the macroscopic and microscopic examination is shown in Tables 1 and 2. During macroscopic dissection, dense adhesions were observed in the Group1 and 3 (Fig. 1). There was a significant difference between the groups when macroscopic adhesions were evaluated $(\mathrm{p}<0.001)$ and the adhesions were significantly fewer in the Groups 2 and 4 (Fig. 2). There was no significant difference between the groups when a degree of inflammation and vascular proliferation were evaluated $(p=0.056, p=0.109)$. Figure 3 shows the histopathologic sections of trachea and mediastinum. There was a significant difference between the groups when foreign body reaction and fibroblasts were 


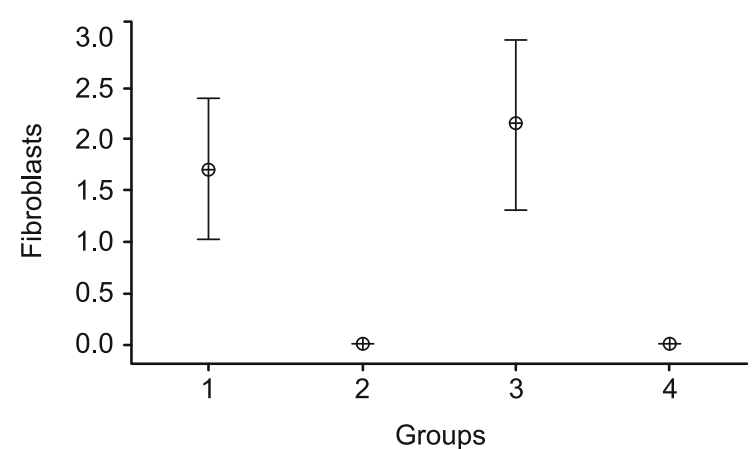

Fig. 4. Interval plot of fibrosis. In Groups 2 and 4,fibroblast proliferation indicating adhesions were significantly less than other Groups.

evaluated $(\mathrm{p}=0.013, \mathrm{p}<0.001)$. Foreign body reaction was significantly lower in the Group 3 when compared to the other groups. Fibroblasts, which indicate adhesions, were lower in the Groups 2 and 4 when compared to the Sham Group and Group 3 (Fig. 4).

\section{Discussion}

In lung cancer, the determination of nodal status is essential for planning therapy and assessing prognosis. For locally advanced lung cancer, re-mediastinoscopy is mainly indicated to evaluate the tumor response in mediastinal lymph nodes after an induction therapy and to indicate further therapy for a definitive pathological proof to show a persistent node invasion i.e. N2 (8). However, mediastinal restaging after the induction therapy remains a difficult and controversial issue although it is technically possible (9-12).

Beginning from 1975, several re-mediastinoscopy series have been reported. Early mediastinoscopy and alternative routes were offered to overcome the adhesions between innominate artery and the trachea (6). It's reported that if the first mediastinoscopy was more complete, more adhesions and fibrosis developed and it was concluded that successful mediastinoscopies might result in a disappointment during re-mediastinoscopy (12). As known, the most serious complication of a mediastinoscopy is major hemorrhage ( $0.4 \%)$ where azygos vein, innominate, pulmonary and bronchial arteries were the most common injured vessels. Despite technical complexity, re-mediastinoscopy still remains as a valuable tool although it has a potentially morbidity (1.9\%) (13-15).

Unfortunately, after recurrent surgical interventions to the same anatomical area, optimal means to prevent adhesions are not available. For this purpose, different agents such as prosthetic barriers, xenograft materials, solutions containing pharmacological agents, bioresorbable membranes, anti-inflammatory drugs have been used. Among them bioresorbable membranes were the most preferred one as it provides a temporary mechanical barrier reducing or preventing the formation of adhesions during tissue repair (16-19).

In this experimental study, we used Repel-cv ${ }^{\circledR}$ as an adhesion barrier. It is a sterile and bioresorbable transparent film comprising of polylactic acid (52 \% LA) and polyethylene glycol (47 \% EO). Its excellent use to reduce the formation of postoperative adhe- sions between pericardium and sternum after cardiac surgery was reported, which directed us to use this barrier in our experimental study. In literature, the mechanism of action is stated as; during the separation of the opposing tissues from each other, it serves to reduce postoperative adhesion formation during the early phase of tissue repair and regeneration $(16,20)$.

Two experimental studies demonstrated the efficacy Repel$\mathrm{CV}^{\circledR}$ in the prevention of retrosternal and pericardial adhesions at time of necropsy at 20 and 21 days $(21,22)$. In a single re-mediastinoscopy rat model by Solak et al (7) it was concluded that, although adhesions were present, anti-adhesive barriers could reduce the difficulties met during re-mediastinoscopy that was performed within a month. However, the late results concerning the efficacy of bioresorbable adhesion barriers to reduce mediastinal adhesions were not investigated. So we decided to test our hypothesis that, late effects of barriers had to be investigated in a model, because in clinical translation, the time period between first and second mediastinoscopy might be postponed and it could exceed one month due to the ongoing chemotherapy.

The most important indicator of adhesion are fibroblasts, which were evaluated and compared between the groups. The results didn't support the efficacy of this bioresorbable adhesion barrier at the second month when used as a single layer, which was not mentioned in any report concerning a re-mediastinoscopy model. However, fortunately when a double layer of same barrier was used, it significantly reduced the mediastinal adhesion formation at the second month similar to single layer used at first month.

Different conclusions were done for the technical practicability of re-mediastinoscopy but it was clear that early re-mediastinoscopy would be much easier. We think that this knowledge would direct further evaluation of the patients for restaging, when re-mediastinoscopy is considered. Although PET might be used in mediastinal restaging, due to its low sensitivity, it is not accepted to be an optimal technique. It was reported that inflammatory reaction occurring after chemoradiotherapy caused a strong accumulation of FDG thus false positive result were seen as high as $20 \%$. Currently, to provide cytohistological information for mediastinal nodal involvement, minimally invasive staging procedures such as endobronchial or endoscopic esophageal ultrasound was offered for the initial intervention. Mediastinoscopy could be performed afterwards and reserved for restaging after induction therapy, and a more difficult remediastinoscopy could be defeated technically $(3,10,23)$. Contrary to this suggestion in one of the largest series of the literature, Marra et al (15) pointed that remediastinoscopy could be done without mortality having a higher sensitivity, specificity and accuracy compared to radiologic investigations, PET/ CT and EBUS/EUS.

Notably, this unique and encouraging experimental study showed that murine models rather than large animals could be used in re-mediastinoscopy studies for further investigations. We think that, the dissection and placement of barrier would be easier and less time consuming in rat models. Another advantage of small animal was that gross pathological analysis could be done concerning the whole trachea and surrounding tissues on the same section. 
As a conclusion, the bioabsorbable membrane composed of polylactic acid and polyethylene glycol used after the initial mediastinoscopy could reduce the difficulties during re-mediastinoscopy, which was performed within a month, and late results were also satisfactory when double layer was used.

\section{References}

1. Olsen PS, Stentoft P, Ellefsen B, Pettersson G. Re-mediastinoscopy in the assessment of resectability of lung cancer. Eur J CardiothoracSurg 1997; 11: 661-663.

2. Bolca C, Sasu AM, Istrate A et al. Remediastinoscopy - current indications. A case report. Pneumologia 2010; 59: 92-94.

3. Van Schil P, Stamatis G. Sensitivity of remediastinoscopy: influence of adhesions, multilevel N2 involvement, or surgical technique? 2006; 24: 5338 .

4. Pitz CCM, Maas KW, Van Swieten HA, Brutel de la Riviere A, Hofman P, Schramel FM. Surgery as a part of combined modality treatment in stage IIIB non-small cell lung cancer. Ann ThoracSurg 2002; 74: 164-169.

5. Meersschaut $D$, Vermassen $F$, Brutel de la Rivière A, Knaepen PJ, Van den Bosch JM, Vanderschueren R. Repeat mediastinoscopy in the assessment of new and recurrent lung neoplasm. Ann ThoracSurg 1992; 53: $120-122$.

6. Palva T, Palva A, Kärjä J. Re-mediastinoscopy. Arch Otolaryngol 1975; 101: 748-750.

7. Solak O, Esme H, Sirmali M et al. Achieving efficient anti-adhesion in remediastinoscopy: an experimental study in rats. Thorac Cardiovasc Surg 2008; 56: 158-161.

8. Rami-Porta R, Call S. Invasive staging of mediastinal lymph nodes: mediastinoscopy and remediastinoscopy. Thorac Surg Clin 2012; 22: 177-189.

9. Rami Porta R. Surgical exploration of the mediastinum by mediastinoscopy, parasternal mediastinotomy and remediastinoscopy: indications, technique and complications. Ann Ital Chir 1999; 70: 867-872.

10. Lardinois D. Pre- and intra-operative mediastinal staging in non-smallcell lung cancer. Swiss Med Wkly 2011; 141: w13168.

11. De Leyn P, Stroobants S, De Wever W, et al. Prospective comparative study of integrated positron emission tomography-computed tomography scan compared with remediastinoscopy in the assessment of residual mediastinal lymph node disease after induction chemothe- rapy for mediastinoscopy-proven stage IIIA-N2 Non-small-cell lung cancer: a Leuven Lung Cancer Group Study. J Clin Oncol 2006; 24: 3333-3339.

12. De Leyn P, Lerut T. Reply to Van Schil and Stamatis Remediastinoscopy: a dangerous tool? Eur J Cardiothorac Surg 2008; 34: 708-709.

13. Park BJ, Flores R, Downey RJ, Bains MS, Rusch VW. Management of major hemorrhage during mediastinoscopy. J Thorac Cardiovasc Surg 2003; 126: 726-731.

14. De Waele M, Hendriks J, Lauwers P, Van Schil P. Different indications for repeat mediastinoscopy: single institution experience of 79 cases. Minerva Chir 2009; 64: 415-418.

15. Marra A, Hillejan L, Fechner S, Stamatis G. Remediastinoscopy in restaging of lung cancer after induction therapy. J Thorac Cardiovasc Surg 2008; 135: 843-849.

16. Haensig M, Mohr FW, Rastan AJ. Bioresorbable adhesion barrier for reducing the severity of postoperative cardiac adhesions: Focus on REPEL-CV(®). Med Devices (Auckl) 2011; 4: 17-25.

17. Stommel MW, Strik C, ten Broek RP, van Goor H. Efficacy and safety of the C-Qur TM film adhesion barrier for the prevention of surgical adhesions (CLIPEUS Trial): study protocol for a randomized controlled trial. Trials 2014; 15: 378.

18. Tchartchian G, Hackethal A, Herrmann A, et al. Evaluation of SprayShieldTM adhesion barrier in a single center: randomized controlled study in 15 women undergoing reconstructive surgery after laparoscopic myomectomy. Arch Gynecol Obstet 2014; 290: 697-704.

19. Hamaji M, Kojima F, Komatsu T, Tsuruyama T, Date H, Nakamura T. A synthetic bioabsorbable sheet may prevent postoperative intrapleural adhesions following thoracotomy: a canine model. Interact CardiovascThorac Surg 2014. Doi: 10.1093/icvts/ivu299.

20. Schreiber C, Boening A, Kostolny $\mathbf{M}$ et al. European clinical experience with REPEL-CV. Expert Rev Med Devices 2007; 4: 291-295.

21. Okuyama N, Wang CY, Rose EA et al. Reduction of retrosternal and pericardial adhesions with rapidly resorbable polymer films. Ann Thorac Surg 1999; 68: 913-918.

22. Okuyama N, Rodgers KE, Wang CY et al. Prevention of retrosternal adhesion formation in a rabbit model using bioresorbable films of polyethylene glycol and polylactic acid. J Surg Res 1998; 78: 118-122.

23. Medford AR, Bennett JA, Free CM, Agrawal S. Mediastinal staging procedures in lung cancer: EBUS, TBNA and mediastinoscopy. Curr Opin Pulm Med 2009; 15: 334-342. 